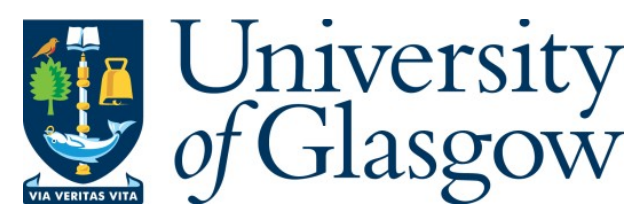

Kappl, M., Kaveh, F., and Barnes, W. J. P. (2016) Nanoscale friction and adhesion of tree frog toe pads. Bioinspiration and Biomimetics, 11(3), 035003.

There may be differences between this version and the published version. You are advised to consult the publisher's version if you wish to cite from it.

http://eprints.gla.ac.uk/129600/

Deposited on: 20 October 2016

Enlighten - Research publications by members of the University of Glasgow http://eprints.gla.ac.uk 
Bioinspiration and Biomimetics 11, (2016) 035003.

\title{
Nanoscale friction and adhesion of tree frog toe pads
}

\author{
Michael Kappl $^{1 *}$, Farzaneh Kaveh ${ }^{1}$, and W. Jon P. Barnes ${ }^{2}$ \\ ${ }^{1}$ MPI for Polymer Research, Mainz, Germany, ${ }^{2}$ Institute of Molecular Cell and Systems Biology, \\ University of Glasgow, UK \\ *kappl@mpip-mainz.mpg.de
}

\begin{abstract}
Tree frogs have become an object of interest in biomimetics due to their ability to cling to wet and slippery surfaces. In this study, we have investigated the adhesion and friction behavior of toe pads of White's tree frog (Litoria caerulea) using atomic force microscopy in aqueous medium. Facilitating special types of AFM probes with radii of $\sim 400 \mathrm{~nm}$ and $\sim 13 \mu \mathrm{m}$, we were able to sense the frictional response without damaging the delicate nanopillar structures of the epithelial cells. While we observed no significant adhesion between both types of probes and toe pads in wet condition, frictional forces under such conditions were very pronounced and friction coefficients amounted between 0.3 and 1.1 for the sliding friction between probes and the epithelial cell surfaces.
\end{abstract}

\section{Introduction}

The toe pads of climbing animals (e.g. geckos and tree frogs) that can adhere rather than just grasp possess a number of features that are absent from most commercial adhesives [1, 2]. In particular, adhesion is not only strong but is also reversible, so that the adhesive surface can be used over and over again. Indeed, attachment and detachment takes place apparently effortlessly every time the animal takes a step. The adhesive surface is additionally self-cleaning, so does not get soiled with normal use. Finally, the adhesive only sticks when required; i.e. particular movements are necessary to produce adhesion so that inadvertently touching a surface does not necessarily result in attachment. Initial experiments were mainly aimed at the study of gecko adhesion given the ease with which geckos can climb walls and run across ceilings [3]. The toe pads of geckos are covered in tiny hairs (setae), which are finely divided, each branch ending in a tiny flat spatula, ca $0.2 \mu \mathrm{m}$ in both length and breadth [4]. Such spatula can make extremely close contact with a surface, so that adhesion is primarily due to van der Waals forces [5]. Such structures are impossible to copy exactly, but arrays of artificial setae have been manufactured (e.g. [6]) and recent designs can carry significant loads up to and including the weight of a human [7]. Tree frogs adhere by a rather different mechanism. They secrete a thin layer of fluid between toe pad and substrate and adhesion is thought to be mainly by capillarity [8, 9], though viscosity-based hydrodynamic forces may also play a role, particularly in torrent/rock frogs that can climb wet rock even when their toe pads are under water [10]. Such a mechanism, that functions on wet as well as dry surfaces, also has implications for biomimetics, for instance in the medical field or in the tire industry. Recently developments include a friction device for safety razors [11] and surgical graspers [12]. 
The distal regions of the digits are enlarged in tree frogs, the ventral parts of these enlargements forming their adhesive toe pads. The specialized toe pad epithelium is surrounded distally and laterally by deep channels that serve to divert water away from the undersurface of each toe pad. The toe pad epithelium itself is a hierarchical structure, where the flat-topped polygonal cells, of which the majority are hexagonal [13], are separated from each other by grooves. Pores of mucus glands end in these channels and produce the fluid that is essential to the toe pads' function. The grooves will help spread the mucus evenly under the pad in dry conditions and help get rid of excess fluid when the surface is wet [14]. Although the surface of each cell is macroscopically flat, microscopically it is covered by a dense array of nanopillars, called pegs by some early authors, though the name is inappropriate as the nanopillars, indeed the whole pad epithelium, has a low elastic modulus [15]. Although adhesion and friction forces measured from individual toe pads with a custom-built force transducer are in the region of $1-5 \mathrm{mN} / \mathrm{mm}^{2}[10,16]$, a recent study of the forces acting on the toes of tree frogs landing on a wooden stick could be as high as fourteen times the body weight of the frogs [17]. This equates to a force of ca $26 \mathrm{mN} / \mathrm{mm}^{2}$, based on a mean force of $0.55 \mathrm{~N}$ and a pad area (2 toes) of $21 \mathrm{~mm}^{2}$ (or as high as $57 \mathrm{mN} / \mathrm{mm}^{2}$ if the frog lands using only a single toe). Insights into the function of different pad structures have also been gained by studying the properties of artificial mimics $[11,18,19]$. However, for dry conditions, adhesion for structured surfaces was generally lower compared to flat surfaces simply due to reduced contact area[19]. For instance, the presence of pillar structures (mimicking the epithelial cells and their surrounding channels) made little difference to adhesive forces under wet conditions, but gave rise to significantly higher friction forces [19, 20]. Higher frictional forces for patterned structures may arise from the increased peeling energies necessary when crack propagation gets arrested at the channels [21, 22].

In this study, the techniques of atomic force microscopy were used to study adhesion and friction at the micro- and nanoscale level. Since, under water, capillarity forces would be absent, any adhesive forces measured with an AFM probe would be due to van der Waals forces. There is good evidence for boundary friction between the tips of the nanopillars and a smooth surface [23]. Indeed, Federle et al's [23] study demonstrated that $41 \%$ of epithelial cells had an average fluid film thickness in the range $0-5 \mathrm{~nm}$. At these distances under water, van der Waals forces might contribute to some extent, but hydrodynamic forces might be more pronounced, unless dry contact is established [24]. Turning to friction, it is surprising that frogs can apparently generate high friction forces, even though they have a fluid joint. Our study was able to measure such forces and locate them with respect to both cell boundaries and nanopillars. They clearly show that the toe pad epithelium of tree frogs is highly adapted to generate high friction forces even in the absence of adhesion.

\section{Experimental methods}

Atomic force microscope (AFM) imaging and force measurements were performed using a Nanowizard AFM (JPK Instruments, Berlin, Germany). Three types of AFM probe were used: 1) standard silicon AFM tips with a nominal tip radius of less than $10 \mathrm{~nm}$ (Olympus OMCL-AC240TS) and spring constants of $1.0-1.4 \mathrm{~N} / \mathrm{m}$ that can be used both for contact and intermittent contact mode imaging in liquids. 2) AFM probes with a tip radius of around $400 \mathrm{~nm}$. These probes were prepared from the same type of cantilevers by heating them to $1200^{\circ} \mathrm{C}$ for $12 \mathrm{~h}$ in air using a high temperature tube furnace (ROC 38/250/16 horizontal, THERMCONCEPT, Bremen, Germany). This leads to additional formation of $\mathrm{SiO}_{2}$ at the silicon surface of the probe, subsequent softening of that layer and rounding off of the tip. The resulting probes had tip radii of around $400 \mathrm{~nm}$. [25] 3) Colloid probes were prepared by attaching silica particles with a nominal radius of $15 \mu \mathrm{m}$ (Duke Scientific, Palo Alto, CA) to tipless silicon cantilevers (NSC12 from NT-MDT, nominal spring constant $7.5 \mathrm{~N} / \mathrm{m}$ ). Vertical spring constants for all cantilevers were determined before measurements using the thermal tune 
method. [26]. Lateral force sensitivity of the AFM probes to measure friction forces were determined using the wedge calibration method [27] using a calibration grating (TGG01 from Mikromasch, Wetzlar, Germany). For the colloid probe, direct calibration with this method was not possible since the colloid probe radius $(13.3 \mu \mathrm{m})$ was much larger than the spacing of the facets of the calibration grating of $3 \mu \mathrm{m}$. We therefore assumed that the friction coefficient for the rounded silica probe on a silicon wafer of 0.3 should be the same as that of the silica colloidal probe on the same wafer. Thus, we measured the load dependence of the lateral deflection signal on a flat silicon wafer for the colloid probe and set the lateral deflection sensitivity such that the slope of the resulting friction signal versus load curve matched the value of 0.3 . All these force sensitivity calibrations were carried out in dry condition. All friction measurements on the toe pads, however, were done under liquid. We therefore corrected for the change in refractive index of the Ringer solution to calculate correct friction forces for deflection signals recorded in liquid [28]. Tip radii of the colloid probe and the rounded off AFM tips were determined by reverse imaging. The probes were mounted in the AFM and used to image a TGT1 calibration grating (NT-MDT) that consists of an array of sharp tips with a tip radius of less than $10 \mathrm{~nm}$. The resulting image is an array of spheres with a radius that corresponds to the probe radius. By exporting the height data and fitting the curvature of the spheres in Origin, we obtained the probe radii. For the rounded off tip used, the radius was $398 \pm 9 \mathrm{~nm}$, while the colloid probe had a radius of $13.3 \mu \mathrm{m}$.

Juvenile White's tree frogs (Litoria caerulea) with a snout-vent length of ca $24 \mathrm{~mm}$ and weighing ca $1.4 \mathrm{~g}$ were killed immediately before the experiments by immersion in a lethal dose of benzocaine in accordance with UK regulations on research using animals. A stock solution of benzocaine was made up by dissolving $1 \mathrm{~g}$ of benzocaine in a mixture of $95 \mathrm{ml}$ of ethanol and $5 \mathrm{ml}$ of water. The frogs were immersed in a solution consisting of $5 \mathrm{ml}$ of stock solution in $100 \mathrm{ml}$ of water until all movement ceased. For the experiments, one of the feet of the frog was held in a small Petri dish $(3.5 \mathrm{~cm}$ in diameter) using Blu-tack. One of the longer digits ( $2^{\text {nd }}$ or $3^{\text {rd }}$ toes of either fore- or hind-limb) was held ventral side uppermost on a small piece of wood, with the toe pad held firm by a pair of staples pressed into the Blu-tack. AFM recordings were made with the whole foot immersed in frog Ringer solution.

Height and friction images were recorded in Ringer solution in contact mode using scan sizes of 25 $\mu \mathrm{m}$ by $25 \mu \mathrm{m}$ and $5 \mu \mathrm{m}$ by $5 \mu \mathrm{m}$ at scan rates of $0.5 \mathrm{~Hz}$. The fast scan direction of the AFM was chosen to be perpendicular to the long axis of the AFM cantilever, in order to simultaneously record topography (height signal) and friction (lateral deflection signal). The height images were flattened by subtracting a second order plane fit from the image to remove overall tilt and curvature of the toe pad. The raw lateral deflection signal images were converted to friction images by subtracting for each image retrace from trace signals and dividing by two. This automatically eliminates a possible offset and its drift of the lateral deflection signal. By multiplying the resulting image data with the lateral force sensitivity using Gwyddion software (http://gwyddion.net, Version 2.40) [29], we obtain quantitative friction image data. Force distance curves on toe pads were recorded in force spectroscopy mode at a scan fixed scan rate of $1 \mathrm{~Hz}$. At least 25 force curves were recorded in each spot. To determine the vertical deflection sensitivity of the cantilever in Ringer solution, force curves were recorded on the glass substrate at the bottom of the petri dish onto which the toe pads had been fixed before and after taking data on the toe pads. To convert raw deflection signal vs. piezo position data into force vs. distance curves, a self-written LabView program was used. 


\section{Results}

AFM imaging with standard AFM tips. AFM imaging of the freshly prepared toe pads in Ringer solution using intermittent contact mode with a standard AFM tip was easily possible and we were able to resolve the characteristic features described in earlier studies (Figure1). The surface of the toe pad is covered by columnar epithelial cells. These roughly hexagonal cells with diameters in the order of 10-15 $\mu \mathrm{m}$ are separated by narrow channel of about $1 \mu \mathrm{m}$ width. The upper surface of the cells is covered by densely packed nanopillars (Figure 1) with a diameter of about 200-400 nm. By stopping the AFM scan and recording force vs. distance curves, one can probe the adhesion and stiffness of the cell surface. We did not observe any adhesion between tip and cell surface upon separation between tip and sample. For a quantitative analysis of the indentation of the cell by the probe, the exact shape of the AFM tip needs to be known, which is hard to access. We therefore did not further evaluate the data taken with these standard AFM tip, but preferred to use probes with a defined tip shape as described below. In order to measure frictional forces between tip and and toe pad surface, we needed to image the cell surfaces in contact mode. To establish a stable contact between tip and sample, we had to use a setpoint in the order of $0.1 \mathrm{~V}$, which corresponded to a normal load between tip and sample of around $10 \mathrm{nN}$. Apparently, the normal and shear forces during scanning under these conditions were too high to allow stable imaging without destruction of the cell surface. During the first scan on a newly selected area, the position of single cells could be identified, but image contrast was low and was usually completely lost during consecutive scans. We were therefore not able to reproducibly record friction forces using standard AFM probes.

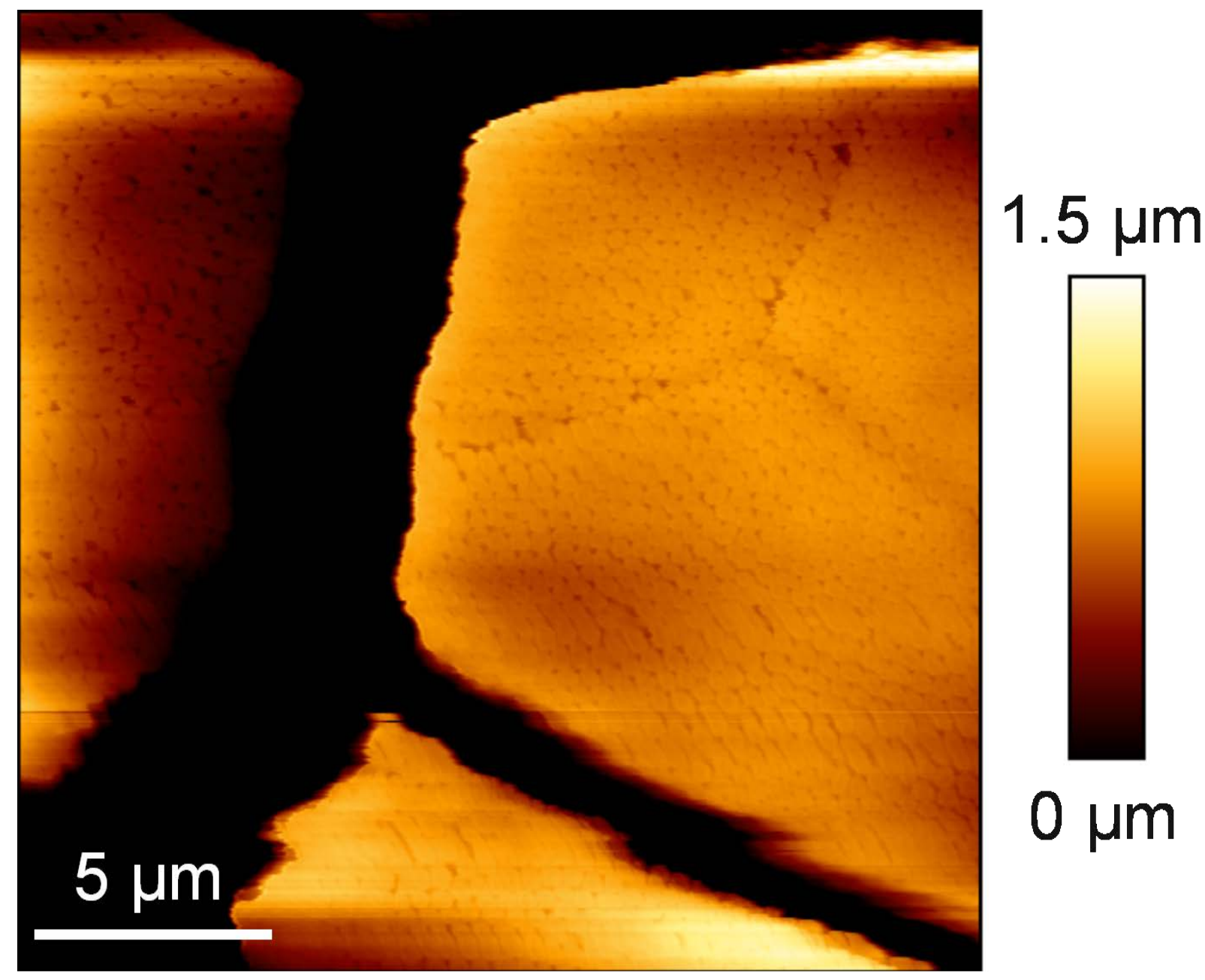


Figure 1: Intermittent contact mode image of the toe pad in Ringer solution. The image shows parts of three toe pad epithelial cells and the channels between them, shown as black because of their depth of several $\mu \mathrm{m}$. Close examination of the surface of the cells shows the closely packed array of nanopillars in surface view. Also visible is a 'scar', indicating the borders of the previous outer cell layer, shed when the frog last moulted.

AFM imaging with large radius AFM tips. To allow for a quantitative analysis of adhesion and deformation data and to reduce local contact pressures, we carried out experiments using AFM probes with significantly larger tip radii. The first type of probe was a silicon AFM that had been rounded off by heat treatment that had a $400 \mathrm{~nm}$ tip radius (398 $\pm 9 \mathrm{~nm}$ ). In spite of the large tip radius, we were still able to get satisfactory image resolution to identify the position of individual epithelial cells. To probe the adhesion between probe and the toe pad surface in Ringer solution, force distance curves were recorded in the center of individual cells. No adhesion could be detected with the $400 \mathrm{~nm}$ tip (Figure 2a), so either no adhesion existed or it was below the detection limit given by the noise level of the zero force baseline, which was typically $100 \mathrm{pN}$. To achieve higher force sensitivity, we also used much larger colloid probe with a radius of $13.3 \mu \mathrm{m}$. In this case, an average adhesion force of $5.0 \mathrm{nN} \pm 1.3 \mathrm{nN}$ was found (Figure $2 \mathrm{~b}$ ). In relation of the probe size, this is a very small value. Using the JKR theory [30], one can relate this adhesion force to the interfacial energy between probe and toe pad by:

$$
F_{a d h}=3 \gamma \pi R
$$

This results in an interfacial energy of only $0.04 \mathrm{mN} / \mathrm{m}$ between colloid probe and cell surface in Ringer solution. The adhesion force measured with the same colloid probe on a dry silicon wafer in air was $4-5 \mu \mathrm{N}$, whereas adhesion on the toe pad after removal of the Ringer solution was too large to be measured $(>100 \mu \mathrm{N})$, since the travel range of the piezo $(15 \mu \mathrm{m})$ was not sufficient to separate the surfaces again after they had been brought into contact.
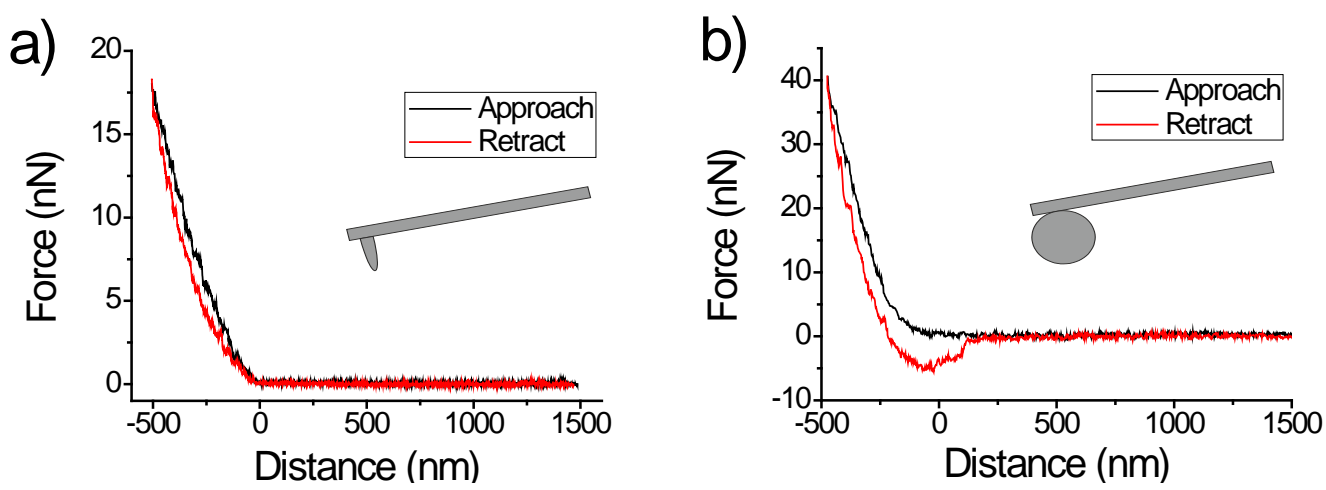

Figure 2: a) Typical force vs. distance curve on a toe pad in Ringer solution recorded with rounded tip ( $\mathrm{r} \approx 400 \mathrm{~nm}$ radius). A Hertz fit of the approach curve gave a reduced elastic modulus of $57 \mathrm{kPa}$. No adhesion between tip and toe pad is observed upon retract. b) as in a) but data recorded with a colloid probe with $13.3 \mu \mathrm{m}$ radius, showing a pull-off force of $5 \mathrm{nN}$.

As there was no pronounced hysteresis between approach and retract force curves, we can assume that the deformation of the epithelial cells is almost fully elastic. We can therefore use the Hertz model [31] of elastic contact between a sphere and a planar surface to obtain the elastic modulus of the toe pad. Since we do not know the value of the Poisson's ratio for the epithelial cells, we only give values of the reduced elastic modulus. From the force curves recorded with the rounded AFM tip with 400 nm radius, we obtained a value of the reduced elastic modulus of $54 \pm 7 \mathrm{kPa}$. Fits to force curves 
recorded with the colloid probe (13.3 $\mu$ m radius) yielded a value for the reduced elastic modulus of $40.7 \pm 3.2 \mathrm{kPa}$.
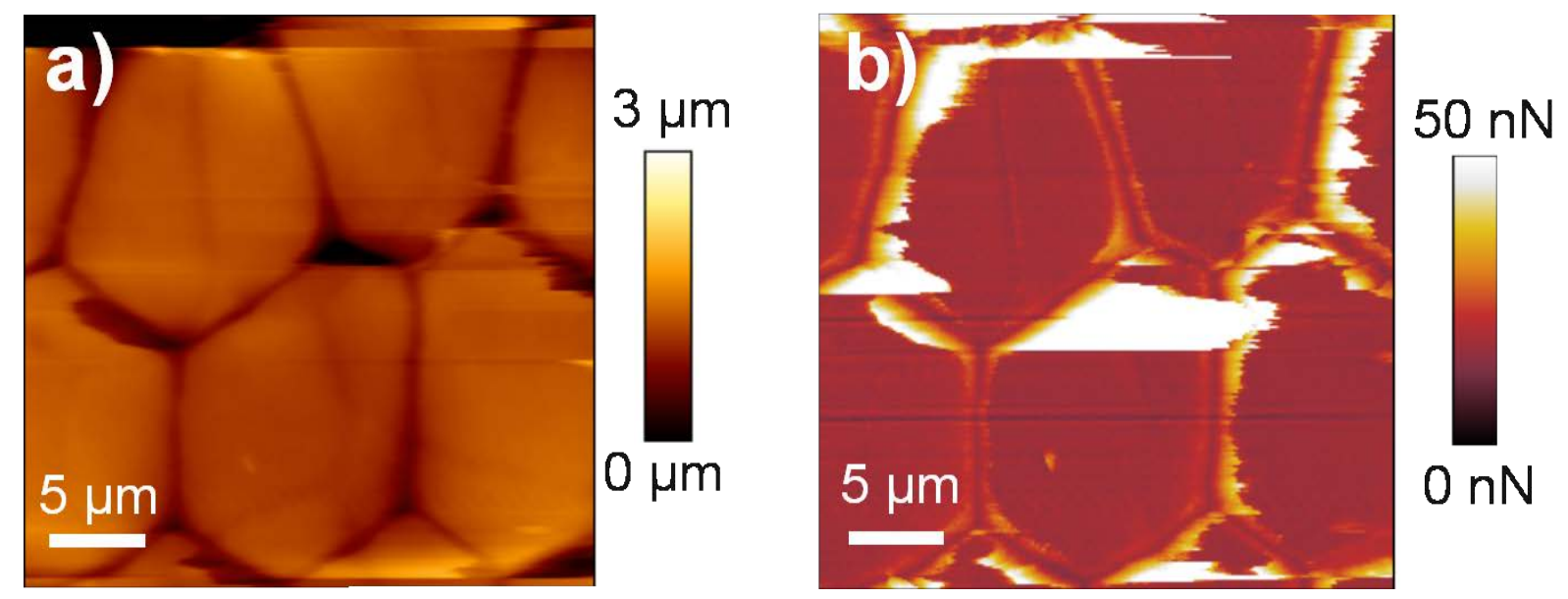

Figure 3: a) contact mode height image and b) friction force image recorded on a toe pad using the 400 nm rounded tip.

By imaging the toe pad surface in contact mode, we were able to record topography and locally resolved friction at the same time. At a scan size of $25 \mu \mathrm{m}$ x $25 \mu \mathrm{m}$, we can clearly identify single epithelial cells with a diameter in the order of 7-15 $\mu \mathrm{m}$ in the height image (Figure 3a). In the corresponding friction image (Figure $3 \mathrm{~b}$ ), we can in addition recognize the presence of nanopillars densely covering the cell surfaces. Highest friction is found always found at the cell borders. In some areas, friction reached maximal values after the tip had passed over the gap between cells, with streaks developing from that point on. This might indicate a pickup of material from the cell edge by the tip which led to higher friction thereafter until the material has been rubbed off again. To exclude the influence of this possible damage at the borders, experiments were carried out on top of single cells without passing over cells edges by zooming in on the corresponding areas with a scan size of $5 \mu \mathrm{m} x$ $5 \mu \mathrm{m}$ (Figure 4). With such a reduced scan area in the center of single cells, we could record many consecutive images without observing wear or surface damage. On that smaller image scale, nanopillars can be resolved also in the height image (Figure 4a). The friction image from the same scan, however, still gives better contrast for the single nanopillars (Figure $4 \mathrm{~b}$ ). Highest values of friction force are observed at the edge of the nanopillars.
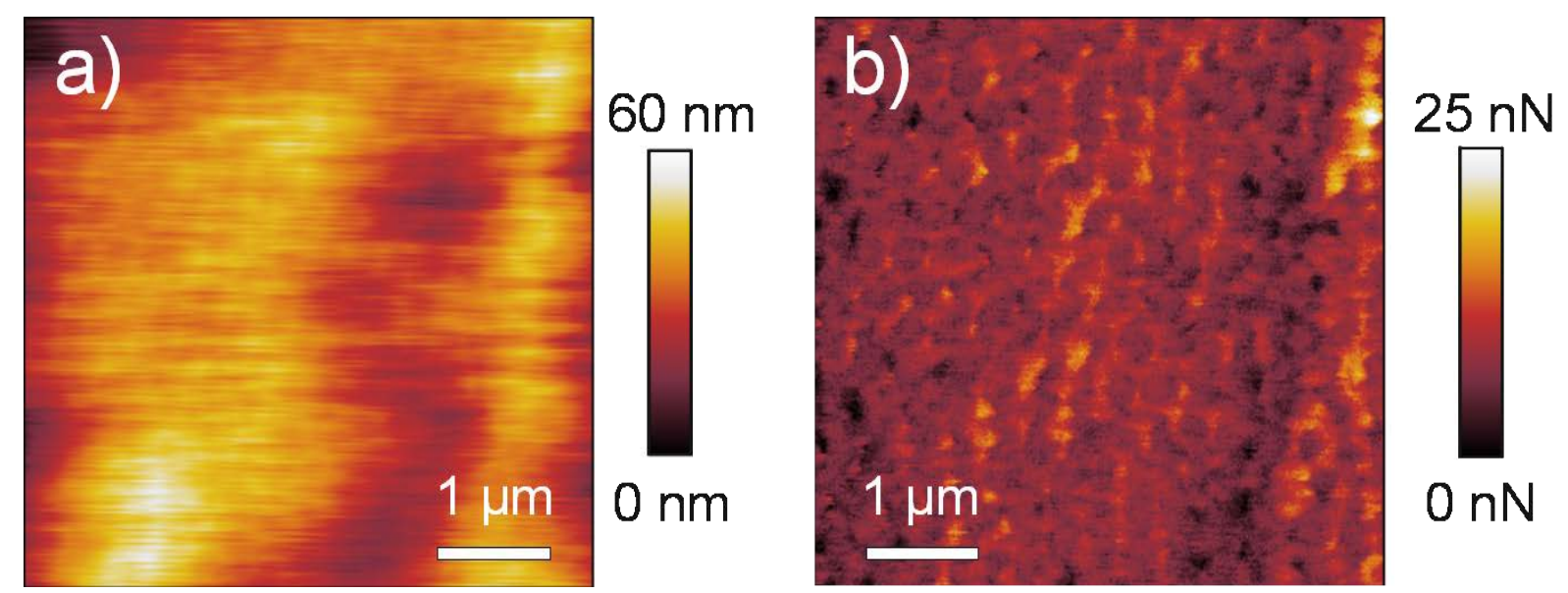

Figure 4: a) height image and b) friction image recorded at the center of a single cell using a rounded tip with $400 \mathrm{~nm}$ radius. 
By varying the applied load on the $400 \mathrm{~nm}$ radius probe by increasing the setpoint value of the AFM while recording the friction images, we were able to determine the load dependence of friction. Therefore, average value and standard deviation of each friction image were calculated using the histogram function of the Gwyddion software. From the observed linear dependence of friction force on applied load (Figure 5), we can deduce a friction coefficient by a linear fit of the data (red lines in Figure $5 \mathrm{a}$ and $\mathrm{b}$ ). When the AFM cantilever was aligned along the proximal-distal axis of the toe pad, which means the scan direction of the probe on the toe pad itself was was perpendicular to that axis, we obtained a friction coefficient of $\mu=1.12 \pm 0.05$. This value was obtained by fitting all data from 3 different cells in Figure 4a simultaneously. Fitting the individual data sets from single cells gave friction coefficients of $1.05,1.13$, and 1.30, respectively. When the orientation of the AFM cantilever was perpendicular to the proximal-distal axis, which means the scan direction of the probe itself was parallel to this axis, we obtained a friction coefficient of $\mu=0.87 \pm 0.02$ as overall fit for the data from six individual cells, with values of for the individual fits to single data sets ranging from 0.81 and 0.95 .
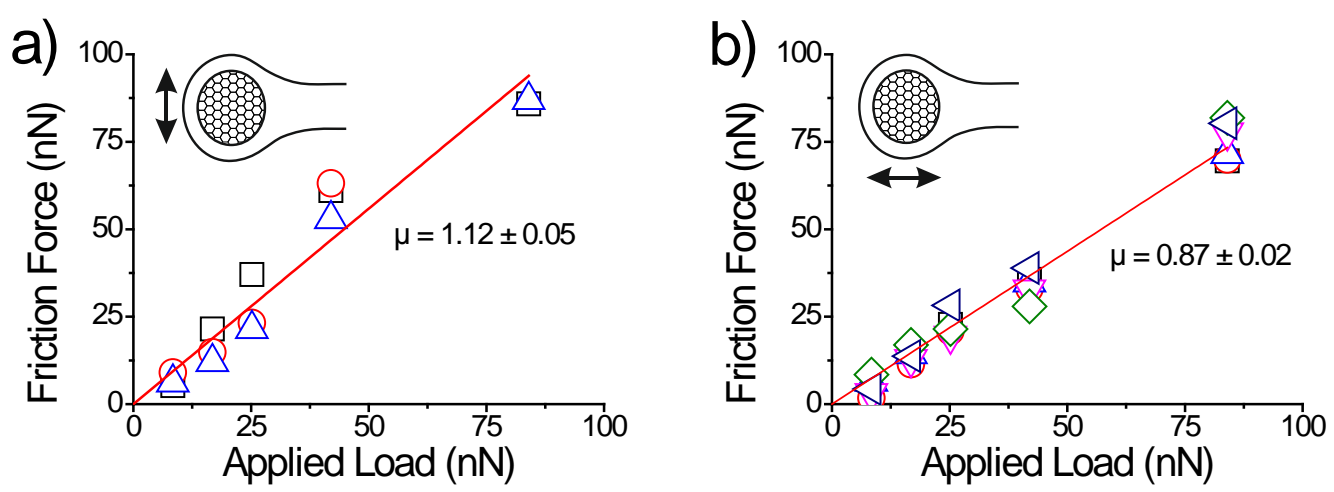

Figure 5: Plots of friction vs. applied load to determine friction coefficients for the $400 \mathrm{~nm}$ probe on toe pads for two perpendicular scan directions. Friction coefficient is the measured friction force divided by applied load, i.e. the slope of a linear fit of the data. Different symbols denote data sets recorded on different cells. Red line is a combined linear fit of all data sets. a) Scan direction perpendicular to toe pad proximal-distal axis, friction coefficient of $\mu=1.12 \pm 0.05$, b) scan direction parallel to proximal-distal axis, friction coefficient of $\mu=0.87 \pm 0.02$.

When imaging the surface of a single cell using the $13.3 \mu \mathrm{m}$ colloid probe, resolution in the height images was poor due to the large probe radius. However, it was still possible to identify the location of single cells. When doing contact mode scans on top of singles cell, details of the structure of the cells surface could no longer be resolved (Figure 6a). The friction image however, still gave a remarkable clear contrast, allowing us to resolve the location of single nanopillars (Figure 6b). 

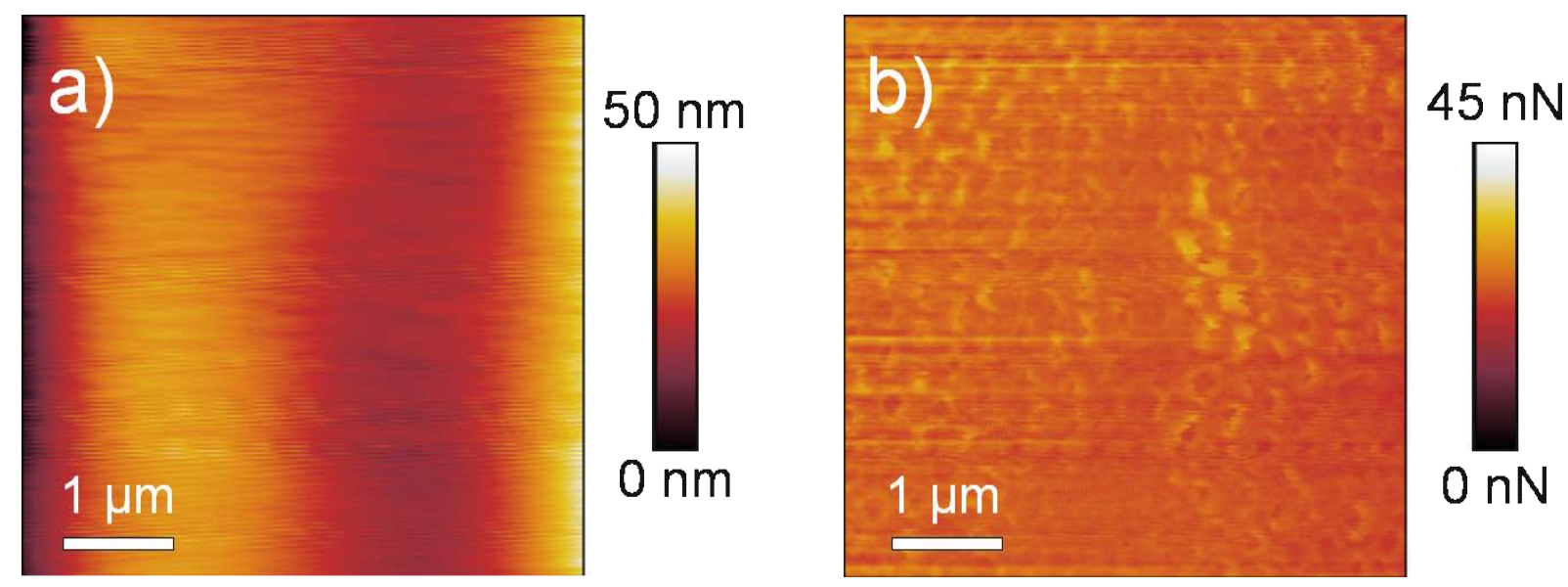

Figure 6: a) Height image and b) friction image recorded on top of a single toe pad epithelial cell using a $13.3 \mu \mathrm{m}$ radius colloid probe. While there is low resolution in the height image due to the large tip radius, the friction image still allows nanopillars to be resolved.

As before, we used friction images recorded at increasing applied loads to study the load dependence of friction also for this larger probe. When the AFM cantilever was aligned along the proximal-distal axis of the toe pad, which means the scan direction of the probe on the toe pad itself was perpendicular to that axis, we obtained a friction coefficient of $\mu=0.45 \pm 0.03$ (Figure 7a). This value was obtained by fitting all data from 3 different cells in Fig. 6 a simultaneously. Fitting the individual data sets from six single cells gave friction coefficients of $0.29,0.35,0.37,0.50,0.54$ and 0.64 , respectively. When the orientation of the AFM cantilever was perpendicular to the proximal-distal axis, which means the scan direction of the probe itself was parallel to this axis, we obtained a friction coefficient of $\mu=0.30 \pm 0.02$ as overall fit for the data from four individual cells, with values of for the individual fits to single data sets of $0.21,0.28,0.29$, and 0.40 , respectively (Figure $7 b$ ). Friction coefficients were thus significantly lower when the larger probe was used.
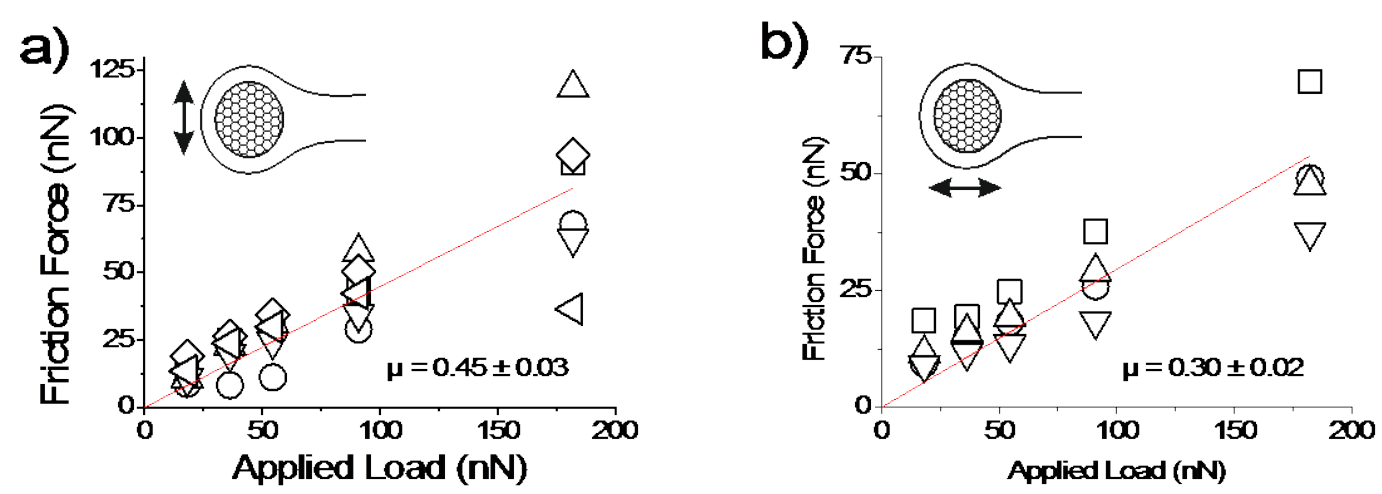

Figure 7: Plots of friction force vs. applied load to determine friction coefficients for the $13.3 \mu \mathrm{m}$ probe on toe pads for two perpendicular scan directions. Different symbols denote data sets recorded on different cells. Red line is a combined linear fit of all data sets. a) Scan direction perpendicular, friction coefficient of $\mu=0.45 \pm 0.03$, b) scan direction parallel to proximal-distal axis, friction coefficient of $\mu=0.30 \pm 0.02$. 


\section{Discussion}

We were able to image the toe pad surfaces of the tree frog, Litoria caerulea,in Ringer solution by AFM using intermittent contact mode, resulting in images that reflect all relevant features of the toe pad structure (Figure 1). The polygonal epithelial cells have a diameter of 10-15 $\mu \mathrm{m}$ and are separated by channels of about $1 \mu \mathrm{m}$ width, that appear black in the color coding of the image due to their depth of several micrometers. The cell surfaces are densely covered by nanopillars with a diameter of 200$400 \mu \mathrm{m}$. The cell partially visible in the upper right part exhibits scars at its surface that originate from the last moult of the toe pad [13]. Our original approach had been to use the same tip after high resolution imaging in tapping mode and switch to contact mode in order to record topography and friction at the same spot. This failed due to normal and shear forces exceeding the stability limit of the epithelial cell surfaces. A possible way to avoid this problem might have been to use much softer AFM cantilevers that could perhaps have allowed stable contact mode imaging with lower contact forces. However, as the aim of our study was to extract quantitative data on both adhesion and friction, we preferred to use probes with a larger, well defined, tip shape and radius. Using probe radii that were orders of magnitude higher than that of standard AFM tip, we were able to reduce contact pressures to an extent that allowed repeated contact image scans without wear of the cell surface. With a known tip radius we could furthermore easily extract the effective elastic modulus of the epithelial cells.

Adhesion between the $400 \mathrm{~nm}$ AFM probe and the toe pad surface in Ringer solution was below the detection limit of around $100 \mathrm{pN}$ given by the noise of the zero force baseline in these experiments (Figure 2a). Using the $13.3 \mu \mathrm{m}$ probe we could resolve an adhesive interaction of around $5 \mathrm{nN}$. Using the JKR model [30] for adhesive contact between a sphere and a flat surface (Eq 1), we obtain an interfacial energy of $0.04 \mathrm{mN} / \mathrm{m}$ between probe and epithelial cell. As the probe and toe pad were fully immersed in Ringer solution, no capillary forces can be present. At the slow approach and retract speeds of a few micrometers per second, hydrodynamic forces will also be negligible. The observed adhesion will be most likely due to van der Waals forces between tip and cell surface, which are lowered in an aqueous environment due to the high dielectric constant of water and are typically one order of magnitude lower than under dry conditions [32]. As both the silica probes and toe pad should be hydrophilic, attractive van der Waals forces may also be partially compensated by repulsive hydration forces [32]. Using the same probe under "dry" conditions i.e. after removing the bulk solution but still having moisture present on the toe pad, adhesion forces of 4-5 $\mu \mathrm{N}$ were observed, that were more than 3 orders of magnitude higher than those in liquid. Previous studies found evidence that adhesion of tree frog to surfaces relies on capillary forces [8, 9], which cannot be present under our experimental conditions in liquid. From interference reflection microscopy experiments it is known that the fluid film between toe pads and contacting surfaces tends to drain quickly down to the nanometer range [23]. This could either lead to formation of a dry contact with increased van der Waals forces or to strong hydrodynamic forces due to the small gap size. Adhesion between toe pad and the $13.3 \mu \mathrm{m}$ probe in ambient conditions (in air but with a humid pad surface) was beyond the measurement range of the AFM/probe combination of about $100 \mu \mathrm{N}$. The absence of any significant adhesion when immersed in liquid and the very pronounced adhesion in "dry" condition is a strong indication that capillary forces are the most likely origin for the macroscopic adhesion of toe pads [8, 9], with possible additional contributions from hydrodynamic forces [23, 24] or van der Waals forces in the case of formation of a dry contact.

Using indentation depths of the order of $500 \mathrm{~nm}$, we found a reduced elastic modulus of around 40-50 $\mathrm{kPa}$ using either the $400 \mathrm{~nm}$ or $13.3 \mu \mathrm{m}$ probe. This value demonstrates the pronounced softness of the material and is in close agreement to results obtained by macroscopic indentation test on toe pads, 
where values of 7-20 $\mathrm{kPa}$ have been found [33]. However, it is partially in contrast to previous AFM indentation experiments with sharp tips, where values between $30 \mathrm{kPa}$ and $65 \mathrm{MPa}$ were found depending on the position within the toe pad [15]. The reason for this discrepancy is not clear. Some source of uncertainty in that earlier study may have been the less well defined tip shape of the sharp AFM tip. Certainly, a second AFM study (13) gave figures much closer to our own, namely a reduced elastic modulus of $33.5 \pm 4.1 \mathrm{kPa}$ (mean $\pm \mathrm{SD}$ ) for Litoria toe pads.

While adhesion forces between probes and cells surfaces were hardly present, friction forces were remarkably high (Figures 5 and 7), leading to friction coefficients between 0.5 and 1 . Such high friction coefficients are mostly found for friction of dry, unlubricated contacts. For our situation, where the probe and toe pad were fully immersed and the liquid is expected to act as lubricant, such high values of the friction coefficient most likely arise from viscous dissipation due to deformation of the soft toe pad surface. Values of the friction coefficient in the order of 1 had also been found before on the whole animal scale [14].

Friction coefficients were consistently lower for the larger probe radius. The mismatch between size scales of the probe and the length scale of nanopillars in the case of the $13.3 \mu \mathrm{m}$ probe might lead to lower friction forces due a less pronounced viscous dissipation due to nanopillar deformation.

Friction was highest at the border of the hexagonal cells (Figure 3b). This can to some extent originate from a larger effective contact area between probe and sample surface, when the probe enters and leaves the channels during scanning. It might, however, also hint to an underlying mechanism to promote formation of dry contact during sliding, where the rim the epithelial cells acts as a kind of rubber lip, that assists drainage of liquid and formation of dry contact [24, 34]. From the contrast in the friction force images (Figure 4b), it is apparent that friction appears to be higher also at the rims of the nanopillars. Since the large probe radius does not allow the probe to penetrate into the channels between the nanopillars, a pure geometric effect can be excluded in this case. Enhancement of friction under wet conditions for microstructured surfaces has been found in model systems before [19, 20], but has not yet been verified for nanoscale systems. The fact that increased friction is also observed at the rim of the nanopillars is strong hint that this type of nanostructure also help to increase wet friction by arrest of crack propagation at the channel edges during sliding [21]. Higher frictional forces for patterned structures may arise from the increased peeling energies necessary when crack propagation gets arrested at the channels. Therefore, the presence of the nanopillars might indeed be a key feature for the outstanding friction performance of the cell surface under wet conditions. This is emphasized by the fact that the nanopillars are still giving a clear contrast in the friction force image (Fig. 6b) even for the $13.3 \mu \mathrm{m}$ probe, while the probe radius is already too large to give any nanoscale resolution of the cell surface topography.

By carrying out friction force experiments with two different scan orientation of the probe, we could observe higher values of the friction coefficient when shearing perpendicular to the proximal-distal axis as compared to the direction along the proximal-distal axis. The exact origin for this directionality of friction is not clear. However, it is known that the cell surface is supported by fibrils that extend vertically from the cell surface in to the cell interior [13]. A possible preferential orientation or tilt of these fibrils could lead to different shear strength and dissipation intensity depending on shearing direction.

\section{Conclusion}

Atomic force microscopy using special probes with defined tip radii allowed us to characterize adhesion and friction of tree frog toe pad epithelial cells on the micro- and nanoscale in the fully 
immersed state. The almost complete absence of adhesion under these conditions is a strong indication that adhesion at the whole animal level is either due to capillary forces or hydrodynamic forces; with some possible contribution of van der Waals forces if dry contacts are formed. Frictional forces are significant even in presence of liquid, giving rise to friction coefficients of $0.5-1$, which are as large as typical values for dry friction. These large values and the fact that the nanopillars give rise to frictional contrast even for a $13.3 \mu \mathrm{m}$ probe radius emphasizes the role of the micro- and nanostructure of the epithelial cells for their exceptional friction performance.

\section{Acknowledgements}

The authors acknowledge funding by priority program SPP1420 of the German Science Foundation and thank Uwe Rietzler for preparing the rounded AFM tips.

1. Creton, C. and S. Gorb, Sticky Feet: From Animals to Materials. MRS Bulletin, 2007. 32(06): p. 466-472.

2. Barnes, W.J.P., Biomimetic Solutions to Sticky Problems. Science, 2007. 318(5848): p. 203204.

3. Autumn, K., How gecko toes stick: the powerful fantastic adhesive used by geckos is made of nanoscale hairs that engage tiny forces, inspiring envy among human imitators. American Scientist, 2006. 94: p. 124-133.

4. Autumn, K. and A.M. Peattie, Mechanisms of Adhesion in Geckos. Integrative and Comparative Biology, 2002. 42(6): p. 1081-1090.

5. Autumn, K., et al., Evidence for van der Waals adhesion in gecko setae. Proceedings of the National Academy of Sciences, 2002. 99(19): p. 12252-12256.

6. Murphy, M.P., et al., Waalbot II: Adhesion Recovery and Improved Performance of a Climbing Robot using Fibrillar Adhesives. The International Journal of Robotics Research, 2010.

7. Hawkes, E.W., et al., Human climbing with efficiently scaled gecko-inspired dry adhesives. Journal of The Royal Society Interface, 2015. 12(102).

8. Emerson, S.B. and D. Diehl, Toe pad morphology and mechanisms of sticking in frogs. Biological Journal of the Linnean Society, 1980. 13(3): p. 199-216.

9. Hanna, G. and W.J.P. Barnes, Adhesion and Detachment of the Toe Pads of Tree Frogs. Journal of Experimental Biology, 1991. 155(1): p. 103-125.

10. Endlein, T., et al., Sticking under Wet Conditions: The Remarkable Attachment Abilities of the Torrent Frog, Staurois guttatus. PLoS ONE, 2013. 8(9): p. e73810.

11. Tsipenyuk, A. and M. Varenberg, Use of biomimetic hexagonal surface texture in friction against lubricated skin. Journal of the Royal Society Interface, 2014. 11(94): p. 20140113.

12. Chen, H., et al., Bioinspired Surface for Surgical Graspers Based on the Strong Wet Friction of Tree Frog Toe Pads. ACS Applied Materials \& Interfaces, 2015. 7(25): p. 13987-13995.

13. Barnes, W.J.P., et al., Comparative Cryo-SEM and AFM studies of hylid and rhacophorid tree frog toe pads. Journal of Morphology, 2013. 274(12): p. 1384-1396.

14. Barnes, W.J.P., C. Oines, and J.M. Smith, Whole animal measurements of shear and adhesive forces in adult tree frogs: insights into underlying mechanisms of adhesion obtained from studying the effects of size and scale. Journal of Comparative Physiology A, 2006. 192(11): p. 1179-1191.

15. Scholz, I., et al., Ultrastructure and physical properties of an adhesive surface, the toe pad epithelium of the tree frog, Litoria caerulea White. Journal of Experimental Biology, 2009. 212(2): p. 155-162.

16. Endlein, T., et al., Sticking like sticky tape: tree frogs use friction forces to enhance attachment on overhanging surfaces. Journal of the Royal Society Interface, 2013. 10(80): p. 1-11. 
17. Bijma, N.N., S.N. Gorb, and T. Kleinteich, Landing on branches in the frog Trachycephalus resinifictrix (Anura: Hylidae). Journal of Comparative Physiology A, 2016: p. 1-10.

18. Huang, C.C., et al., The effects of humidity and surface free energy on adhesion force between atomic force microscopy tip and a silane self-assembled monolayer film. Journal Of Materials Research, 2010. 25(3): p. 556-562.

19. Drotlef, D.-M., et al., Insights into the Adhesive Mechanisms of Tree Frogs using Artificial Mimics. Advanced Functional Materials, 2013. 23(9): p. 1137-1146.

20. Varenberg, M. and S.N. Gorb, Hexagonal Surface Micropattern for Dry and Wet Friction. Advanced Materials, 2009. 21(4): p. 483-486.

21. Ghatak, A., et al., Peeling from a biomimetically patterned thin elastic film. Proceedings of the Royal Society of London. Series A: Mathematical, Physical and Engineering Sciences, 2004. 460(2049): p. 2725-2735.

22. Chung, J.Y. and M.K. Chaudhury, Roles of discontinuities in bio-inspired adhesive pads. Journal of the Royal Society Interface, 2005. 2(2): p. 55-61.

23. Federle, W., et al., Wet but not slippery: boundary friction in tree frog adhesive toe pads. Journal of the Royal Society Interface, 2006. 3(10): p. 689-697.

24. Persson, B.N.J., Wet adhesion with application to tree frog adhesive toe pads and tires. Journal of Physics: Condensed Matter, 2007. 19(37): p. 376110.

25. Hüttl, G., et al., Tailored colloidal AFM probes and their TEM investigation. Surface and Interface Analysis, 2002. 33: p. 50-53.

26. Hutter, J.L. and J. Bechhoefer, Calibration of atomic-force microscope tips. Review of Scientific Instruments, 1993. 64(7): p. 1868-1873.

27. Varenberg, M., I. Etsion, and G. Halperin, An improved wedge calibration method for lateral force in atomic force microscopy. Review of Scientific Instruments, 2003. 74(7): p. 3362-3367.

28. Tocha, E., et al., Calibration of Friction Force Signals in Atomic Force Microscopy in Liquid Media. Langmuir, 2007. 23: p. 7078-7082.

29. Nečas, D. and P. Klapetek, Gwyddion: an open-source software for SPM data analysis. Central European Journal of Physics, 2012. 10(1): p. 181-188.

30. Johnson, K.L., K. Kendall, and A.D. Roberts, Surface Energy and the Contact of Elastic Solids. Proceedings of the Royal Society of London A: Mathematical, Physical and Engineering Sciences, 1971. 324(1558): p. 301-313.

31. Hertz, H., Über die Berührung fester elastischer Körper, in Journal für die reine und angewandte Mathematik. 1882. p. 156.

32. Butt, H.J., K. Graf, and M. Kappl, Physics and Chemistry of Interfaces. 3rd ed. 2013, Heidelberg: Wiley VCH.

33. Barnes, W.J.P., P. Perez-Goodwyn, and S.N. Gorb, Mechanical properties of the toe pads of the tree frog, Litoria caerulea. Comparative Biochemistry and Physiology. Part A: Molecular and Integrative Physiology, 2005. 141: p. 145.

34. Barnes, W.J.P., et al., Bionics and wet grip. Tire Technol. Int., 2002. December 2002: p. 56-60. 